\title{
Derivation of a planetary ionospheric storm index
}

\author{
T. L. Gulyaeva ${ }^{1,2}$ and I. Stanislawska ${ }^{2}$ \\ ${ }^{1}$ IZMIRAN, 142190 Troitsk, Moscow Region, Russia \\ ${ }^{2}$ Space Research Centre PAS, Bartycka 18-A, 00-716 Warsaw, Poland
}

Received: 30 June 2008 - Accepted: 5 August 2008 - Published: 11 September 2008

\begin{abstract}
The planetary ionospheric storm index, $W_{p}$, is deduced from the numerical global ionospheric GPS-IONEX maps of the vertical total electron content, TEC, for more than half a solar cycle, 1999-2008. The TEC values are extracted from the 600 grid points of the map at latitudes $60^{\circ} \mathrm{N}$ to $60^{\circ} \mathrm{S}$ with a step of $5^{\circ}$ and longitudes $0^{\circ}$ to $345^{\circ} \mathrm{E}$ with a step of $15^{\circ}$ providing the data for 00:00 to $23: 00 \mathrm{~h}$ of local time. The local effects of the solar radiant energy are filtered out by normalizing of the TEC in terms of the solar zenith angle $\chi$ at a particular time and the local noon value $\chi_{0}$. The degree of perturbation, DTEC, is computed as log of TEC relative to quiet reference median for 27 days prior to the day of observation. The $\mathrm{W}$-index map is generated by segmentation of DTEC with the relevant thresholds specified earlier for $f_{o} \mathrm{~F} 2$ so that 1 or -1 stands for the quiet state, 2 or -2 for the moderate disturbance, 3 or -3 for the moderate ionospheric storm, and 4 or -4 for intense ionospheric storm at each grid point of the map. The planetary ionospheric storm $W_{p}$ index is obtained from the W-index map as a latitudinal average of the distance between maximum positive and minimum negative $\mathrm{W}$-index weighted by the latitude/longitude extent of the extreme values on the map. The threshold $W_{p}$ exceeding 4.0 index units and the peak value $W_{p} \max \geq 6.0$ specify the duration and the power of the planetary ionosphere-plasmasphere storm. It is shown that the occurrence of the $W_{p}$ storms is growing with the phase of the solar cycle being twice as much as the number of the magnetospheric storms with $D_{s t} \leq-100 \mathrm{nT}$ and $A_{p} \geq 100 \mathrm{nT}$.
\end{abstract}

Keywords. History of geophysics (Solar-planetary relationships) - Space plasma physics (Experimental and mathematical techniques; Instruments and techniques)

Correspondence to: T. L. Gulyaeva

(gulyaeva@izmiran.ru)

\section{Introduction}

Over the last decade an extensive database of GPS-derived total electron content (TEC) measurements has become available from both space- and ground-based observations, and these measurements have been used to establish the general morphology of the global TEC distribution (Davies and Hartmann, 1997). Vertical TEC represents an integral plasma density in the column of $1 \mathrm{~m}^{2}$ in the ionosphere and plasmasphere from $65 \mathrm{~km}$ to $20200 \mathrm{~km}$ at the GPS satellites' altitude over the Earth.

The quiet ionospheric state is characterized by the regular diurnal, seasonal, spatial and solar cycle changes of electron density, or electron content, according to the energy input from the UV/EUV radiation of the Sun: sunlight conditions at different geodetic locations, the relation between geodetic and geomagnetic coordinates, the state of the magnetosphere and neutral atmosphere. Regular quiet time variations of the ionosphere have been reproduced by the global electron content, GEC, giving a single value from the sum of the TEC over the world (Afraimovich et al., 2008).

In contrast to the planetary geomagnetic indices (Menvielle and Berthelier, 1991), the planetary index of the ionospheric perturbations has not been established up to now since it is difficult to immediately deduce from a mixture of increases and decreases of plasma density and electron content on the globe (Ho et al., 1996; Zhao et al., 2007). The ionospheric irregularities are superimposed on the quiet state variations showing positive deviation (TEC or electron density increases) or negative deviation (TEC decreases) relative to the quiet reference at local, regional or global scale (Mendillo, 2006). To avoid two-fold symptoms of TEC deviations, the standard deviation of TEC was used by Jakowski et al. (2006) in the regional segments for direct comparisons with the planetary geomagnetic index of a dominant sign - positive for $A_{a}, A_{p}, K_{p}$, but negative for $D_{s t}$ index. Special GPS-derived irregularity metrics in terms of the

Published by Copernicus Publications on behalf of the European Geosciences Union. 


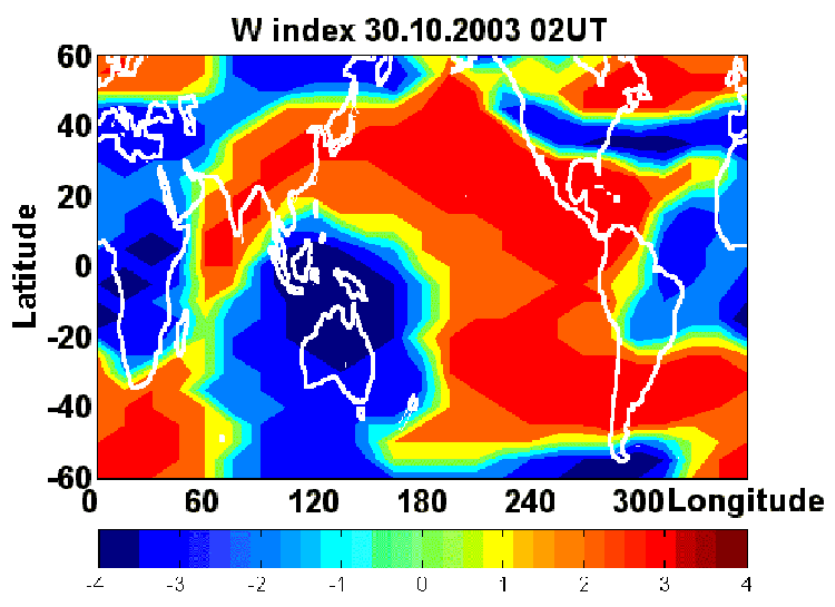

Fig. 1. Map of positive and negative ionospheric indices during the peak of the ionospheric super-storm on 30 October 2003 derived from the TEC global map.

goodness-of-fit parameter of TEC mapping, allows ranking the regional ionospheric storms with $D_{s t}$ storms in the magnetosphere (Sparks et al., 2007). Development of the planetary index for the ionosphere perturbations would be helpful for identifying the ionospheric storms occurring under magnetically quiet-time conditions (Mikhailov et al., 2007).

We have used global ionospheric maps of total electron content, GIM, provided by the Jet Propulson Laboratory (JPL) in the IONEX format (Manucchi et al., 1998; Schaer et al., 1998) as the database for deriving the planetary index of the ionospheric storms. To filter out differences in the diurnal/seasonal contribution of the solar ionizing radiation, the vertical TEC has been normalized by a factor related to the solar zenith angle $\chi$ at a particular time and the local noon value $\chi_{0}$ (Gulyaeva, 2008). Similar to the ionospheric peak plasma density, $N m \mathrm{~F} 2$, the proxy for TEC coincides with the observation at noon but it is gradually reduced from day to night. Multiplying the TEC by the coefficient $C$ $\left(\chi, \chi_{0}\right)$ makes proxy for TEC varying more homogeneously throughout the year, exhibiting similarity of a diurnal profile at all seasons. This reduction improves the correlation between the data at different locations and improves the interseasonal correlation for data of any particular location on the globe. Further analysis is applied to the proxy for TEC reduced by the solar zenith angle denoted as "TEC" for the simplicity.

\section{Database and index derivation}

The global GPS-JPL maps of the total electron content provide numerical representation of the TEC over the world at latitudes of $87.5^{\circ} \mathrm{S}$ to $87.5^{\circ} \mathrm{N}$ in step of $2.5^{\circ}$, longitudes from $180^{\circ} \mathrm{W}$ to $180^{\circ} \mathrm{E}$ in step of $5^{\circ}$ with temporal resolution of $2 \mathrm{~h}$ UT. In view of a huge volume of data processed (27 daily- hourly running maps for median calculation at 12 two-hourly UT pixels for the period of 1999-2008), the number of spatial grid points of each map was reduced to 600 grids per map embracing the planetary surface within the closed field lines: latitudes from $60^{\circ} \mathrm{S}$ to $60^{\circ} \mathrm{N}$ in step of $5^{\circ}$ (number of latitudes $n=25$ ), longitudes from $180^{\circ} \mathrm{W}$ to $180^{\circ} \mathrm{E}$ in step of $15^{\circ}$ (number of longitudes $m=24$ ) providing local time coverage from 00:00 to 23:00 LT in step of $1 \mathrm{~h}$.

The decimal logarithm of the hourly value of the TEC, relative to the quiet reference TECmed, is taken as a measure of TEC perturbation at each grid point of the map (Gulyaeva et al., 2008):

\section{DTEC $=\log ($ TEC $/$ TECmed $)$}

The sign of DTEC specifies the positive or negative phase of the ionospheric perturbation.

A sliding reference, TECmed, is defined as the timecorresponding median of 27 days preceding a day of observation. We assume that the period of 27 days, corresponding to the solar rotation, yields the median value that might also be valid for the 28 th day.

Starting with the segmentation of the ionospheric variability at grid points of the TEC map similar to the F2 layer peak electron density, we apply the thresholds given in Table 3 by Gulyaeva et al. (2008) to the results of the DTEC calculation with Eq. (1). Index $W= \pm 1$ stands for the quiet state, $W= \pm 2-$ the moderate disturbance, $W= \pm 3$ - the moderate ionospheric storm, and $W= \pm 4$ - the intense ionospheric storm.

Immediately, maps of positive and negative ionospheric $\mathrm{W}$-index have been generated with the above algorithm from GPS-IONEX TEC maps for 1999-2008. These maps show the mixture of positive and negative ionospheric perturbations at different regions of the globe as shown in Fig. 1. Since the occurrence of positive and negative indices depends on season, location, external forces, intensity and local starting time of a perturbation, we take the advantage of a round-the-world longitudinal presentation ( $24 \mathrm{~h} \mathrm{LT}$ ) of the index at the latitude which allows the capturing of the ionospheric storm onset at any local time dominant by night (Zhao et al., 2007).

The difference (span) between the maximum of positive index, $W \max j$, and the minimum of negative index, $W \min j$, at the $j$-th latitude, serves as a latitudinal measure of the storm if there are values corresponding to the storm at the particular latitude:

$\delta W j=W \max j-W \min j$ for $W \max j \geq 3$ and/or $W \min j \leq-3$

$\delta W j=\max (W \max j,|W \min j|)$ for $W \max j \leq 2$ and $W \min j \geq-2$

The planetary $W_{p}$ index depicts contributions of perturbation at a global scale:

$W_{p}=\left(1+k n^{-1} m^{-1}\right) n^{-1} \Sigma \delta W j,(j=1, \ldots, n)$

$W_{p}$ is the latitude averaged span between extremes $\delta W j$ at each latitude. The weight $\left(1+k n^{-1} m^{-1}\right)$ in Eq. (2) explains 

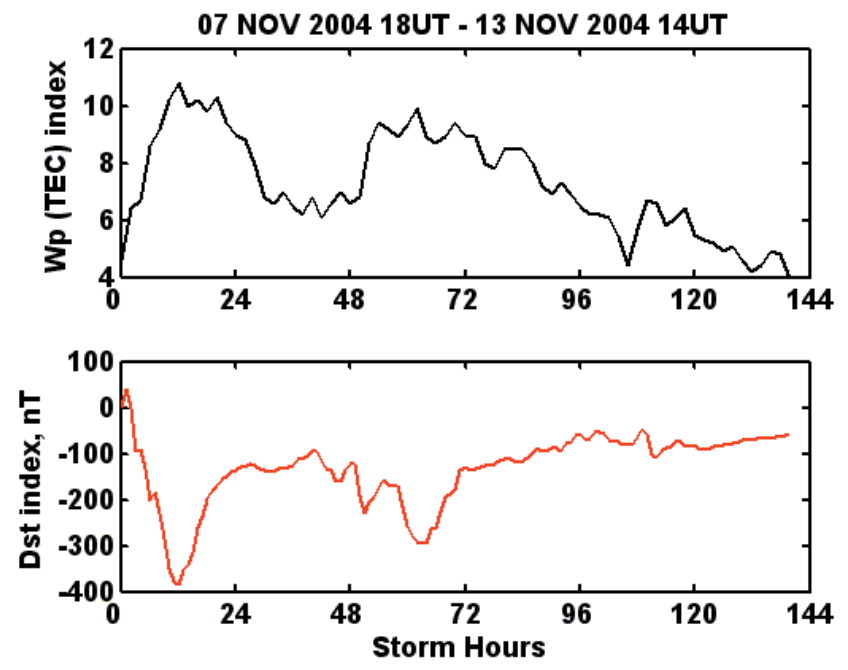

Fig. 2. Coherent ionospheric $W_{p}$ index and magnetospheric $D_{s t}$ index during space weather super-storm on 7 to 13 November 2004.

the latitude-longitude extent of the areas of the greatest positive index $W \max =\max (W \max j)$, and the least negative value $W \min =\min (W \min j)$. Count $k$ represents the number of the extreme positive and negative index values on a map.

\section{Results}

The single-sign positive planetary $W_{p}$ index varying typically from 2 to 12 index units (as different from individual positive and negative markers of $\mathrm{W}$-index from -4 to +4 for each grid point) has been obtained daily for 12 selected UT hours during 1999-2008. Two-hours UT steps, according to GPS-IONEX maps, investigate the events longer than $2 \mathrm{~h}$ in duration. Daily mean and monthly mean values are also produced. The annual listing of $W_{p}$ index for 1999 up to present is provided at http://www.izmiran.ru/services/iweather/storm with the hourly UT and daily mean values including linear interpolation of $2 \mathrm{~h} W_{p}$ index for $24 \mathrm{~h} \mathrm{UT}$ in a step of $1 \mathrm{~h}$ per day.

We define the onset of the planetary ionosphereplasmasphere storm at $W_{p} \geq 4.0$ (the end of the storm when $W_{p}<4.0$ ) which refers to the occurrence of grid storm signatures $(|W j| \geq 3)$ at appreciable number of points $k$ from 30 to more than 400 grid points observed on the $\mathrm{W}$-index map during the peak of the super-storm (Eq. 2). The intensity of $W_{p}$ storm is defined by the peak value $W_{p} \max \geq 6.0$ index units. The power of the storm is defined as the peak value multiplied by the storm duration, in hours. The power greater than 1000 p.u. (power units) has specified 7 ionosphereplasmasphere super-storms for the period analyzed.

With the above criteria, we have identified 160 ionosphereplasmasphere storms for the years between 1999-2008 listed at http://www.izmiran.ru/services/iweather/storm. For a comparison, 69 intense magnetospheric storms with

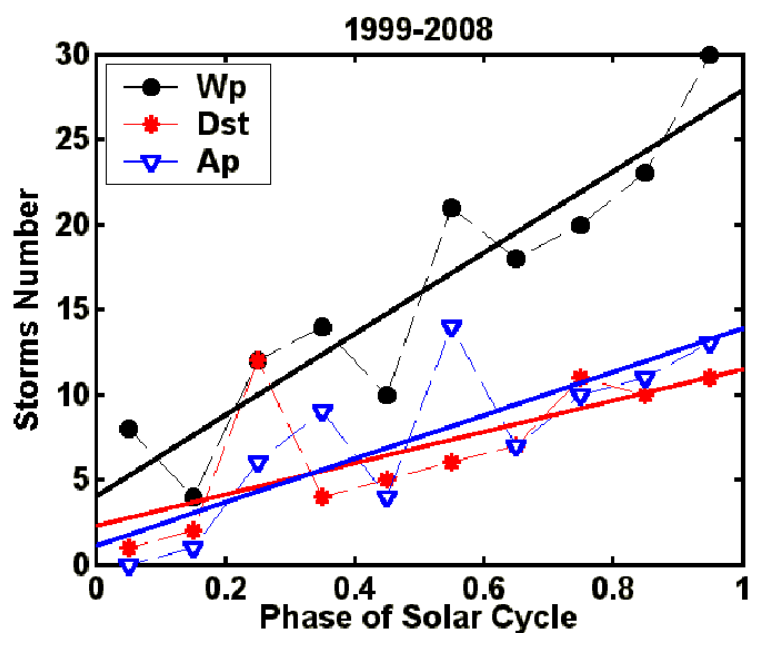

Fig. 3. Solar cycle trend of the number of magnetospheric and plasmaspheric-ionospheric space weather storms observed during 1999-2008.

$D_{s t} \leq-100 \mathrm{nT}$ and 79 storms with $A_{p} \geq 100 \mathrm{nT}$ have occurred during the same period. An example of a coherent ionospheric-plasmaspheric super-storm with Power $=1534$ p.u. and magnetospheric $D_{s t}$ storm is presented in Fig. 2. It demonstrates the advantage of introducing the planetary index depicting the span between the positive and negative ionospheric indices in a unique planetary index $W_{p}$ (Eq. 2).

Figure 3 presents the occurrence of ionosphereplasmasphere storms and magnetospheric storms versus the phase of the solar cycle. The general trend of a growing storm number can be expressed as

$Y=a+b \Phi$

in terms of the phase of the solar cycle:

$\Phi=(T-m) /(M-m)$

where $T$ is the month of a storm event, $m$ is the month of the solar minimum, $M$ is the month of the solar maximum (the pair of $m$ and $M$ embracing an event). Coefficients of the Eq. (3) are given in Table 1. The coefficients are obtained by the least square fit to the number of storms calculated in bins of $0.1 \Phi$ occurred for the period since 1999 up to present. Figure 3 and Table 1 demonstrate that the ionosphere-plasmasphere storms occur twice as much as the magnetospheric storms.

\section{Summary}

Availability of GPS-derived TEC-IONEX maps since 1998 proved to be an important source for derivation of TEC-based planetary index of ionospheric activity. The TEC values are extracted at 600 grid points of the map at latitudes $60^{\circ} \mathrm{N}$ to 
Table 1. Coefficients of model representing storms number in terms of the phase of the solar cycle.

\begin{tabular}{ccc}
\hline$Y$ & $a$ & $b$ \\
\hline$W_{p}$ & 4.06 & 23.87 \\
$D_{s t}$ & 2.32 & 9.15 \\
$A_{p}$ & 1.11 & 12.79 \\
\hline
\end{tabular}

$60^{\circ} \mathrm{S}$ with a step of $5^{\circ}$ and longitudes $0^{\circ}$ to $345^{\circ} \mathrm{E}$ with a step of $15^{\circ}$ providing hourly values for 00:00 to 23:00 of local time for a representative set of conditions during 19992008. The local effects of solar radiant energy are filtered out by normalizing the TEC in terms of the solar zenith angle $\chi$ at a particular time and the local noon value $\chi_{0}$. The derivation of the planetary perturbation index is a two-step process. In step 1 , the degree of perturbation is computed as a logarithm of TEC relative to quiet reference taken as dailyhourly median for 27 days prior the day of observation. The $\mathrm{W}$-index map is generated by the segmentation of perturbations with relevant thresholds so that $\mathrm{W}$-index is equal to \pm 1 for the quiet state, \pm 2 for the moderate disturbance, \pm 3 for the moderate ionospheric storm, and \pm 4 for the intense ionospheric storm. The sign of $\mathrm{W}$-index refers to the positive or negative phase of the ionospheric perturbation at each grid point of the map. In step 2 , the planetary $W_{p}$ index is obtained from the $\mathrm{W}$-index map as latitudinal average of ranges between maximum positive index and minimum negative index weighted by the latitude/longitude extent of the extreme index values on the map.

The single-sign $W_{p}$ index varies from 2 to 12 index units. Based on the hourly estimate of the planetary $W_{p}$ index, the ionospheric-plasmaspheric storm is identified at $W_{p} \geq 4.0$ with $W_{p} \max \geq 6.0$. The trend of growing number of ionospheric storms towards the solar maximum is expressed by linear equation in terms of the phase of the solar cycle. Comparing it with a similar expression obtained for a number of magnetospheric storms with $D_{s t} \leq-100 \mathrm{nT}$ and $A_{p} \geq 100 \mathrm{nT}$ we obtained that the ionospheric storms have occurred twice as much as the magnetospheric storms. Thus, the planetary $W_{p}$ index reveals that the ionospheric storms, which belong specifically to the ionosphere-plasmasphere system, provide a broader proxy index by influencing space weather more than the geomagnetic indices alone.
Acknowledgements. GPS-IONEX maps used in the present study are provided by JPL online at ftp://cddisa.gsfc.nasa.gov/pub/gps/ products/ionex/.

Topical Editor M. Pinnock thanks F. Arikan for her help in evaluating this paper.

\section{References}

Afraimovich, E. L., Astafieva, E. I., Zhivetiev, I. V., Oinats, A. V., and Yasikevich, Yu. V.: Global electron content during solar cycle 23, Geomagnetism Aeronomy, 48, 187-200, 2008.

Davies, K. and Hartmann, G. K.: Studying the ionosphere with the Global Positioning System, Radio Sci., 32, 1695-1703, 1997.

Gulyaeva, T. L.: Proxy for the ionospheric peak plasma density reduced by the solar zenith angle, Earth, Planets, Space, in press, 2008.

Gulyaeva, T. L., Stanislwska, I., and Tomasik, M.: Ionospheric weather: cloning missed $f o \mathrm{~F} 2$ observations for derivation of variability index, Ann. Geophys., 26, 315-321, 2008.

Ho, C. M., Manucci, A. J., Lindquister, U. J., Pi, X., and Tsurutani, B. T.: Global ionosphere perturbations monitored by the worldwide GPS network, Geophys. Res. Lett., 23, 3219-3222, 1996.

Jakowski, N., Stankov, S. M., Schlueter, S., and Klaehn, D.: On developing a new ionospheric perturbation index for space weather operations, Adv. Space Res., 38, 2596-2600, 2006.

Manucci, A. J., Ho, C. M., and Lindqwister, U. J.: A global mapping technique for GPS-driven ionospheric TEC measurements, Radio Sci., 33(8), 565-582, 1998.

Mendillo, M.: Storms in the ionosphere: patterns and processes for total electron content, Rev. Geophys., 47, RG4001, 1-47, 2006.

Menvielle, M. and Berthelier, A.: The K-derived planetary indices: description and availability, Rev. Geophys., 29, 415-432, 1991.

Mikhailov, A. V., Depueva, A. H., and Depuev, V. H.: Daytime F2-layer negative storm effect: what is the difference between storm-induced and Q-disturbance events?, Ann. Geophys., 25, 1531-1541, 2007.

Schaer, S., Gurtner, W., and Feltens, J.: IONEX: The ionosphere map exchange format Version 1. Proc. IGS AC Workshop, Darmstadt, Germany, 233-247, 1998.

Sparks, L., Komjathy, A., and Manucci, A. J.: Ranking the ionosphere variability, Proc. BSS Symposium, Boston College, Boston, MA, USA, 2007.

Zhao, B., Wan, W., Liu, L., and Mao, T.: Morphology in the total electron content under geomagnetic disturbed conditions: results from global ionospheric maps, Ann. Geophys., 25, 1555-1569, 2007. 